\title{
Experimental Consequences of Family Unification
}

\author{
Jonathan Bagger \\ Lyman Laboratory of Physics, Harvard University, Cambridge, Massachusetts 02138, and Stanford Linear Accelerator Center, \\ Stanford University, Stanford, California 94305 \\ and \\ Savas Dimopoulos \\ Department of Physics, Stanford University, Stanford, California 94305
}

and

Eduard Massó(a) and M. Hall Reno

Stanford Linear Accelerator Center, Stanford University, Stanford, California 94305

(Received 16 April 1985)

\begin{abstract}
Theories of family unification predict four left-handed and four right-handed families of quarks and leptons, all with masses below $265 \mathrm{GeV}$. The lightest mirror quark has a mass of less than 140 $\mathrm{GeV}$. All charged leptons are lighter than $55 \mathrm{GeV}$, and the lightest is below $40 \mathrm{GeV}$. All five new neutrinos have masses less than $40 \mathrm{GeV}$ and contribute to the width of the $Z^{0}$. We study the decays of these new families, and discuss rare processes such as $\mu \rightarrow e \gamma$. We also examine proton decay, and show that it can proceed into $e^{+} \pi^{0}$ at the observable but acceptable rate of $10^{32 \pm 1} \mathrm{yr}$.
\end{abstract}

PACS numbers: $12.10 . \mathrm{En}, 14.60 . \mathrm{Gh}, 14.60 . \mathrm{Jj}, 14.80 . \mathrm{Dq}$

The discovery of the $W$ and $Z$ bosons at CERN has confirmed that the standard $\mathrm{SU}(3) \otimes S U(2) \otimes U(1)$ model provides an excellent description of the strong, weak, and electromagnetic interactions. However, the standard model is in no sense a fundamental theory. It does not explain why there are three forces, nor why the weak interactions are purely $V-A$. It does not tell us why quarks and leptons come in families, nor why the families repeat.

Family-unified theories provide a natural answer to these questions. ${ }^{1-4}$ They preserve the successful features of ordinary grand unified theories, and moreover, they explain the multiplicity of families. In family-unified theories, the forces and the families are both incorporated into irreducible representations of a simple gauge group $G$. The most appealing theories of family unification are based on the group $\mathrm{O}(18)$. All the known families fit into just one representation, the 256-dimensional spinor. This spinor is complex, and so superheavy masses for ordinary fermions are forbidden. Furthermore, the group $\mathrm{O}(18)$ is anomaly free, so that the spinor anomalies cancel among themselves.

Previous attempts to construct theories based on O(18) were plagued by serious difficulties. ${ }^{1}$ These stem from the fact that the 256-dimensional spinor contains eight left- and eight right-handed families. With sixteen light families, the color coupling blows up at a few hundred teraelectronvolts. These theories are not perturbatively unifiable.

To avoid this problem, it is necessary to split the $\mathrm{O}(18)$ spinor and give some families mass at the unification scale $M_{\mathrm{GUT}}$. This was done in Ref. 2 , where it was shown that $\mathrm{O}(18)$ must break to $\mathrm{SU}(3)$ $\otimes \mathrm{SU}(2) \otimes \mathrm{U}(1) \otimes Z_{N}$, where $Z_{N}$ is a discrete symmetry. The $Z_{N}$ family symmetry allows half the leftand right-handed families to gain mass at $M_{\mathrm{GUT}}$. It protects the other four left- and four right-handed families from acquiring mass all the way down to the weak scale $M_{W}$.

The possible $Z_{N}$ symmetries are seriously constrained by cosmology and low-energy phenomenology. In Ref. 4 we studied the restrictions that arise from proton decay, big-bang nucleosynthesis, lefthanded Kobayashi-Maskawa mixing, and the stability of right-handed matter. We discovered that family charges of the fermions are essentially unique, and that $N$ equals five or ten. We found that the lowenergy theory has two Higgs doublets and precisely three ultralight left-handed neutrinos. The extra families of $O(18)$ give rise to dramatic experimental signatures, both for proton decay and for present and upcoming particle accelerators. These signatures are the subject of this Letter.

Right-handed masses.-Since O(18) predicts both left- and right-handed families in the low-energy world, it must explain why the right-handed families are heavier than their left-handed counterparts. It does this via $O(8)$ group theory, which ensures that the Weinberg-Salam Higgs doublets can be chosen to couple only to right-handed families. The righthanded families receive direct masses at the weak scale $M_{W}$, while the masses for the left-handed families are generated by one-loop radiative corrections.

Because of the $Z_{N}$ family charges, only one of the two Higgs doublets couples to the right-handed families. (We denote this field by $\phi$.) Its Yukawa couplings must be rather large for the induced left-handed 
masses to be in accord with experiment. As in any grand unified theory, the Yukawa couplings are specified at the unification scale $M_{\mathrm{GUT}}$. The physical righthanded masses are then obtained by evolving of the Yukawa couplings to low energies by use of the SU(3) $\otimes S U(2) \otimes U(1)$ renormalization-group equations. When the Yukawa couplings are large, the low-energy masses are given by the infrared fixed points of the renormalization-group equations. The details of the masses do not depend on the Yukawa couplings at the unification scale $M_{\mathrm{GUT}}$.

In $O(18)$ the essential features of the right-handed masses are given by this fixed-point behavior. We find the low-energy masses by generating uniform distributions for the up, down, electron, and neutrino Yukawa couplings at $M_{\mathrm{GUT}}$, with values chosen randomly from the interval 1.0-10.0. We then evolve all Yukawa couplings to low energies. The results are collected in Fig. 1, where separate histograms display the up, down, electron, and neutrino Yukawa couplings at the weak scale $M_{W}$. The fixed-point behavior is evident in the peaking of the distributions. Since $\langle\phi\rangle \leqslant 175 \mathrm{GeV}$, we find the following upper bounds on the quark and lepton masses:

$$
\begin{aligned}
& M_{U} \lesssim 265 \mathrm{GeV}, \quad M_{D} \lesssim 230 \mathrm{GeV}, \\
& M_{N} \lesssim 40 \mathrm{GeV}, \quad M_{E} \lesssim 55 \mathrm{GeV} .
\end{aligned}
$$

It is instructive to examine the sums of the Yukawa couplings for each of the sets of initial conditions. ${ }^{4}$ We find that the sums obey $\Sigma_{U} g_{U}^{2} \leqslant 4.0, \Sigma_{D} g_{D}^{2} \leqslant 2.6$, $\Sigma_{N} g_{N}^{2} \leqslant 0.16$, and $\Sigma_{E} g_{E}^{2} \leqslant 0.16$. These bounds give upper limits on the lightest right-handed quarks and leptons:

$$
\begin{aligned}
& M_{U} \leqq 175 \mathrm{GeV}, \quad M_{D} \leq 140 \mathrm{GeV}, \\
& M_{N} \leqq 40 \mathrm{GeV}, \quad M_{E} \leq 40 \mathrm{GeV} .
\end{aligned}
$$

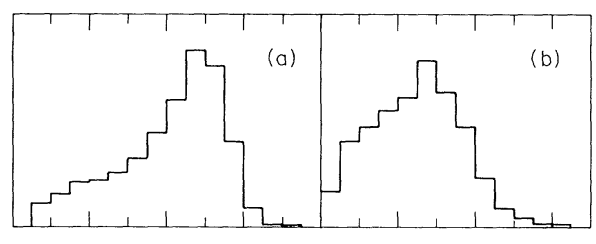

$g_{u}$

$g_{D}$

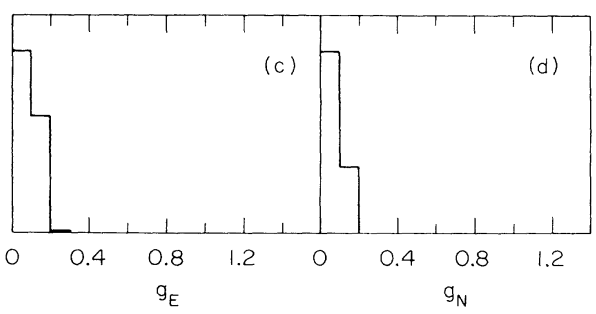

FIG. 1. Histograms of 4000 right-handed (a) up, (b) down, (c) electron, and (d) neutrino Yukawa couplings, evaluated at the weak scale $M_{W}=80 \mathrm{GeV}$.
The bounds (1) and (2) are very stringent. They imply that all four right-handed neutrinos and at least one charged lepton should contribute to the width of the $Z^{0}$. Furthermore, they tell us that at least one right-handed lepton doublet should be seen in the decays of the $W$.

Decays of new families. - The most striking feature of $\mathrm{O}$ (18) family unification is that it predicts five new families below the weak scale. We now summarize the decays of these new families.

The heavier of the eight right-handed quarks cascade to their lighter partners by standard chargedcurrent processes, with lifetimes on the order of $10^{-21}$ sec. If kinematically allowed, the lightest right-handed quarks decay into left-handed quarks by dimensionfive operators,

$$
Q \rightarrow q+\text { scalars, }
$$

with lifetimes of the order of a second. Otherwise, the Cabibbo mixing of right- and left-handed quarks allows decays into real and virtual $W$ bosons,

$$
Q \rightarrow q+W, \quad Q \rightarrow q+f+\bar{f}^{\prime},
$$

with lifetimes on the order of 10 and $10^{4} \mathrm{sec}$, respectively. ${ }^{5}$ The processes (3) and (4) ensure that no stable right-handed matter is seen today.

The renormalization-group analysis discussed above implies that at least one of the four right-handed charged leptons should contribute to the widths of the $W$ and $Z^{0}$. All of the right-handed charged leptons decay by ordinary weak interactions with lifetimes on the order of $10^{-19} \mathrm{sec}^{5}$

The four right-handed neutrinos all have masses less than half that of the $Z^{0}$. The heavier right-handed neutrinos decay into lighter charged leptons by ordinary charged-current processes. The lighter righthanded neutrinos mix with their left-handed partners. Because of this mixing, the light right-handed neutrinos decay into ordinary matter. The lifetimes for all of these decays are of the order of $10^{-16}[(10 \mathrm{GeV}) /$ $\left.M_{N}\right]^{5}$ sec. $^{5}$ If $M_{N}$ is large enough, the right-handed neutrinos contribute to the visible decays of the $Z^{0}$.

Last but not least, $O(18)$ also predicts a new lefthanded family, somewhat lighter than its right-handed counterparts. Its neutrino has mass less than $40 \mathrm{GeV}$, and decays into standard fermions through ordinary charged-current processes. Depending on its lifetime, the fourth left-handed neutrino might contribute to the visible decays of the $Z^{0}$.

Flavor violation.-Two-Higgs models have the potential for large flavor-changing neutral currents through tree-level Higgs boson exchange. $O(18)$ naturally avoids these problems because one Higgs doublet couples primarily to up-type quarks, and the other to down-type quarks. This leads to a suppression of Higgs-mediated $K^{0}-\bar{K}^{0}$ mixing. 
Additional flavor violations occur in the lepton sector. Both the charged and neutral currents induce violations of electron, muon, and tau number. These effects are not necessarily suppressed by $M_{W} / M_{\mathrm{GUT}}$. Charged-current violations occur because of Cabibbo mixings between the three ultralight left-handed neutrinos. These mixings are induced by the Dirac mass of the fourth left-handed neutrino.

Neutral-current flavor violations arise because the light left-handed singlet neutrino has the same family charge as its right-handed counterparts. ${ }^{4}$ The left- and right-handed singlets mix and induce further mixings between the left- and right-handed neutrino doublets. Since left- and right-handed families have opposite weak couplings, these mixings induce direct flavor violations in the couplings of the $Z^{0}$. Such off-di- agonal couplings can give rise to monojet events at CERN and the Stanford linear collider. ${ }^{6}$

The exact mixings of the neutrino states depend on details of the family assignments and lepton mass matrices. In Table I we list the combinations of lefthanded doublets that can mix with the mirror doublets, subject to certain assumptions, for various SU(5) and anti-SU(5) embeddings. ${ }^{7}$ One of these combinations joins with the fourth left-handed singlet to gain a Dirac mass. Note that the conventional SU(5) version is somewhat more restricted than anti-SU(5).

Violations of lepton number are known to be very small. The most stringent limits on our model come from the process $\mu \rightarrow e \gamma$. Its branching ratio has an experimental upper bound of $2 \times 10^{-10} .^{8}$ In $\mathrm{O}(18)$, the dominant contribution to this process is given by a

TABLE I. Neutrino mixings and nucleon decay channels for different SU(5) and antiSU(5) embeddings. The neutrino combinations listed here have Dirac masses and mix with the mirror doublets. The value of $\epsilon$ is of order 0.1 . We include nucleon decay modes induced by $\mathrm{SU}(5)$ and $\mathrm{O}(10)$ gauge bosons. Decays into other channels are suppressed by quark mixing angles.

\begin{tabular}{|c|c|c|c|}
\hline Embedding & Neutrino mixing & $p$ decay modes & $n$ decay modes \\
\hline \multirow[t]{2}{*}{ SU (5) } & $\nu_{\tau}+\epsilon \nu_{\tau^{\prime}}$ & $e^{+} \pi^{0}, \mu^{+} K^{0}$ & $e^{+} \pi^{-}, \bar{\nu}_{e} \pi^{0}$ \\
\hline & & $\bar{\nu}_{e} \pi^{+}, \bar{\nu}_{\mu} K^{+}$ & $\bar{\nu}_{\mu} K^{0}$ \\
\hline \multirow[t]{2}{*}{ SU(5) } & $\nu_{\tau}+\epsilon \nu_{\tau^{\prime}}$ & $\mu^{+} \pi^{0}, \bar{\nu}_{\mu} \pi^{+}$ & $\mu^{+} \pi^{-}, \bar{\nu}_{\mu} \pi^{0}$ \\
\hline & $\nu_{\tau^{\prime}}+\epsilon \nu_{e}$ & & \\
\hline \multirow[t]{2}{*}{ Anti-SU(5) } & $\nu_{\tau}+\epsilon \nu_{\tau^{\prime}}$ & $e^{+} \pi^{0}, \mu^{+} K^{0}$ & $e^{+} \pi^{-}, \bar{\nu}_{e} \pi^{0}$ \\
\hline & & $\bar{\nu}_{e} \pi^{+}, \bar{\nu}_{\mu} K^{+}$ & $\bar{\nu}_{\mu} K^{0}$ \\
\hline \multirow[t]{3}{*}{ Anti-SU(5) } & $\nu_{\tau^{\prime}}$ & $\bar{\nu}_{\tau^{\prime}} \pi^{+}, \bar{\nu}_{\tau} K^{+}$ & $\bar{\nu}_{\tau^{\prime}} \pi^{0}, \bar{\nu}_{\tau} K^{0}$ \\
\hline & $\nu_{\tau}+\epsilon \nu_{\mu}$ & & \\
\hline & $\nu_{\tau^{\prime}}+\epsilon \nu_{\tau}$ & & \\
\hline \multirow[t]{2}{*}{ Anti-SU(5) } & $\nu_{\tau}+\epsilon \nu_{\mu}$ & $e^{+} \pi^{0}, \bar{\nu}_{e} \pi^{+}$ & $e^{+} \pi^{-}, \bar{\nu}_{e} \pi^{0}$ \\
\hline & & $\bar{\nu}_{\tau} K^{+}$ & $\bar{\nu}_{\tau} K^{0}$ \\
\hline \multirow[t]{3}{*}{ Anti-SU(5) } & $\nu_{\tau^{\prime}}+\epsilon \nu_{\mu}$ & $\mu^{+} K^{0}, \bar{\nu}_{\mu} K^{+}$ & $\bar{\nu}_{\mu} K^{0}, \bar{\nu}_{\tau^{\prime}} \pi^{0}$ \\
\hline & $\nu_{\tau}+\epsilon \nu_{e}$ & $\bar{\nu}_{\tau^{\prime}} \pi^{+}$ & \\
\hline & $\nu_{\tau^{\prime}}$ & & \\
\hline \multirow[t]{2}{*}{ Anti-SU(5) } & $\nu_{\tau}+\epsilon \nu_{\tau^{\prime}}$ & $\mu^{+} \pi^{0}, \bar{\nu}_{\mu} \pi^{+}$ & $\mu^{+} \pi^{-}, \bar{\nu}_{\mu} \pi^{0}$ \\
\hline & $\nu_{\tau^{\prime}}+\epsilon \nu_{e}$ & & \\
\hline \multirow[t]{2}{*}{ Anti-SU(5) } & $\nu_{\tau^{\prime}}+\epsilon \nu_{\tau}$ & $\bar{\nu}_{\tau^{\prime}} \pi^{+}$ & $\bar{\nu}_{\tau^{\prime}} \pi^{0}$ \\
\hline & $\nu_{\tau}+\epsilon \nu_{\mu}$ & & \\
\hline
\end{tabular}


$W$ loop, where the internal fermion is one of the Dirac neutrinos $N$. This contribution to the branching ratio is given by ${ }^{9}$

$$
\begin{aligned}
R_{B}(\mu & \rightarrow \text { e } \gamma) \\
& \simeq 2 \times 10^{-7}\left[M_{N} /(10 \mathrm{GeV})\right]^{4}\left|\theta_{\mu}^{*} \theta_{e}\right|^{2},
\end{aligned}
$$

where $\theta_{l}$ denotes the Cabibbo mixing between the lepton $l$ and the neutrino $N$. The suppression of $\mu \rightarrow e \gamma$ restricts the range of neutrino mixing angles. For the extreme case $M_{N} \simeq 40 \mathrm{GeV}$, it implies $\left|\theta_{\mu}^{*} \theta_{e}\right|<10^{-3}$. When $\nu_{e}$ mixes with heavy neutrinos, $\left|\theta_{\mu}^{*} \theta_{e}\right|$ is estimated to be of order $10^{-3}$. Thus some versions of our model predict that $\mu \rightarrow e \gamma$ might soon be seen.

The present limits on other rare processes such as $K_{L} \rightarrow \mu e, \mu \rightarrow e e e, \tau \rightarrow \mu \gamma$, and $\tau \rightarrow e \gamma$ do not impose any further constraints on the theory. Even an improvement on the upper limit of $R_{B}\left(K_{L} \rightarrow \mu e\right)$ to $10^{-12}$ would only imply $\left|\theta_{\mu}^{*} \theta_{e}\right|>0.1$ for $M_{N} \simeq 40$ $\mathrm{GeV}$.

Proton decay.-Limits on the proton lifetime exclude a large class of grand unified theories. O(18), however, escapes this fate. Eight families survive down to low energies, and so the color beta function is dominated by its two-loop contribution. With eight families, $M_{\mathrm{GUT}}$ increases from its standard $\mathrm{SU}(5)$ value and prolongs the proton lifetime. ${ }^{10}$

For $\Lambda_{\overline{\mathrm{MS}}} \simeq 150 \mathrm{MeV}$, the $\mathrm{O}(18)$ proton lifetime is a factor of 900 times the minimal SU(5) prediction, and $\sin ^{2} \theta_{W} \simeq 0.215$. This gives a lifetime on the order of $10^{32 \pm 1}$ yr. $^{4}$ Since experiments now measure $\tau(p$ $\left.\rightarrow e^{+} \pi^{0}\right) \geq 2 \times 10^{32} \mathrm{yr}^{11} \mathrm{O}(18)$ predicts that proton decay could soon be seen.

Nucleon decay channels depend on how quarks and leptons are assigned to $\mathrm{O}(10)$ multiplets. In ordinary grand unified theories, these assignments are not unique. Unconventional choices can lead to nonstandard decay modes. This ambiguity is lessened in the $\mathrm{O}(18)$ theory because of the $Z_{N}$ family symmetry. The form of the fermion mass matrices is known, and unambiguous family assignments can be made for all the quarks on the basis of nearest-neighbor Cabibbo mixing. The lepton family assignments are somewhat more uncertain; but subject to certain assumptions, they permit us to make precise predictions of the nucleon decay channels.

In Table I we have listed the neutrino mixing and nucleon decay modes for each of the possible family assignments. Most of the assignments give compar- able branching ratios for protons into neutrinos and charged leptons. This should be contrasted with supersymmetry, ${ }^{12}$ where kaons and muon neutrinos are favored over charged leptons. Note, however, that supersymmetry mimics those versions of our theory where modes with charged leptons are Cabibbo suppressed.

It is a pleasure to thank Howard Georgi, Sheldon Glashow, Lawrence Hall, Ann Nelson, Stuart Raby, and Robert Wagoner for helpful discussions. This work was supported by the Department of Energy, under Contract No. DOE-AC03-76SF00515, and by the National Science Foundation, under Contracts No. NSF-PHY-83-10654 and No. NSF-PHY-82-15249. One of us (S.D.) acknowledges receipt of an Alfred P. Sloan fellowship and another of us (E.M.) a Fulbright fellowship.

(a) On leave of absence from Departament de Física Teòrica, Universitat Autònoma de Barcelona, Bellaterra, Spain.

${ }^{1}$ M. Gell-Mann, P. Ramond, and R. Slansky, in Supergravity, edited by $P$. van Nieuwenhuizen and D. Freedman (North-Holland, Amsterdam, 1979); F. Wilczek and A. Zee, Phys. Rev. D 25, 553 (1982).

2J. Bagger and S. Dimopoulos, Nucl. Phys. B244, 247 (1984).

${ }^{3}$ G. Senjanović, F. Wilczek, and A. Zee, Phys. Lett. 141B, 389 (1984).

4J. Bagger, S. Dimopoulos, E. Massó, and H. Reno, Stanford Linear Accelerator Center Report No. SLAC-PUB3441,1984 (to be published).

5 One version of the model has two long-lived righthanded quarks and a long-lived lepton, either charged or neutral. The lifetimes of these particles are on the order of $1-10^{7} \mathrm{sec}$.

${ }^{6}$ L. Hall, J. Kim, and A. Nelson, Harvard University Report No. HUTP-85/A020, 1985 (to be published).

${ }^{7}$ S. Barr, Phys. Lett. 112B, 219 (1982); H. Georgi, unpublished.

8J. Bowman et al., Phys. Rev. Lett. 42, 556 (1979).

${ }^{9}$ B. Lee, S. Pakvasa, R. Shrock, and H. Sugawara, Phys. Rev. Lett. 17, 937 (1977).

10J. Bagger, S. Dimopoulos, and E. Massó, Phys. Lett. 145B, 211 (1984).

${ }^{11}$ R. Bionta et al., Phys. Rev. Lett. 51, 27, 522(E) (1983).

12S. Dimopoulos, S. Raby, and F. Wilczek, Phys. Lett. 112B, 133 (1982); J. Ellis, D. Nanopoulos, and S. Rudaz, Nucl. Phys. B202, 43 (1982). 